\title{
Écologie industrielle, économie de la fonctionnalité, positionnements et perspectives communes
}

Industrial Ecology, Economy of Functionality, Common Positions and

Perspectives

Cyril Adoue et Laurent Georgeault

(2) OpenEdition

Journals

Édition électronique

URL : http://journals.openedition.org/developpementdurable/10219

DOI : 10.4000/developpementdurable.10219

ISSN : 1772-9971

Éditeur

Association DD\&T

Référence électronique

Cyril Adoue et Laurent Georgeault, «Écologie industrielle, économie de la fonctionnalité,

positionnements et perspectives communes », Développement durable et territoires [En ligne], Vol. 5, $n^{\circ}$

1 | Février 2014, mis en ligne le 04 février 2014, consulté le 20 avril 2019. URL : http://

journals.openedition.org/developpementdurable/10219; DOI : 10.4000/developpementdurable.10219

Ce document a été généré automatiquement le 20 avril 2019

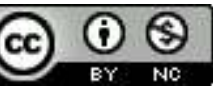

Développement Durable et Territoires est mis à disposition selon les termes de la licence Creative Commons Attribution - Pas d'Utilisation Commerciale 4.0 International. 


\title{
Écologie industrielle, économie de la fonctionnalité, positionnements et perspectives communes
}

\author{
Industrial Ecology, Economy of Functionality, Common Positions and
}

Perspectives

Cyril Adoue et Laurent Georgeault

1 Alors que les vingt ans du Développement Durable viennent d'être célébrés, cet article propose un éclairage sur les relations des pratiques issues du champ de l'écologie industrielle. En effet, au-delà de la définition du champ scientifique que constitue l'écologie industrielle, une perspective historique sur ses déclinaisons opérationnelles (éco-conception, symbioses, économie de la fonctionnalité) permet de situer ces éléments les uns par rapport aux autres.

\section{L'avènement de l'écologie industrielle, pour une croissance durable}

2 L'écologie industrielle est née aux États-Unis. Deux évènements marquent cet avènement. Le premier est la publication par Frosch et Galopoulos en 1989 dans Scientific American d'un article intitulé "Strategies for manufacturing" associant pour la première fois les termes écologie et industrielle (Frosch et Gallopoulos, 1989). Cette publication est suivie de la thèse de Brad Allenby en 1992 (Allenby, 1992) qui théorise l'écologie industrielle et propose une méthode d'éco-conception.

Dans un premier temps, l'écologie industrielle intègre un questionnement aux dimensions essentiellement environnementale et économique.

Elle introduit un cadre conceptuel nouveau qui, grâce à une approche systémique empruntée à l'écologie scientifique, permet de dresser un diagnostic sur les relations complexes entre la société industrielle contemporaine et les autres écosystèmes de la 
biosphère. Elle s'interroge sur la limitation des pollutions industrielles, la nécessité d'optimiser les procédés industriels, la recherche de réutilisation de sous-produits. Frosch et Gallopoulos introduisent l'analogie du système industriel avec le fonctionnement des écosystèmes naturels tout en étant conscients des limites du modèle proposé. Il s'agit de la recherche d'une éco-efficacité, d'une éco-efficience. Allenby, en 1992, propose une thèse dans laquelle il redéfinit une typologie des systèmes industriels en considérant leurs relations avec la biosphère (Allenby, 1992). L'écologie industrielle vise à tendre vers le type III de sa typologie, i.e. un système uniquement alimenté par des ressources renouvelables (figure 1).

Figure 1 : Évolution du système industriel
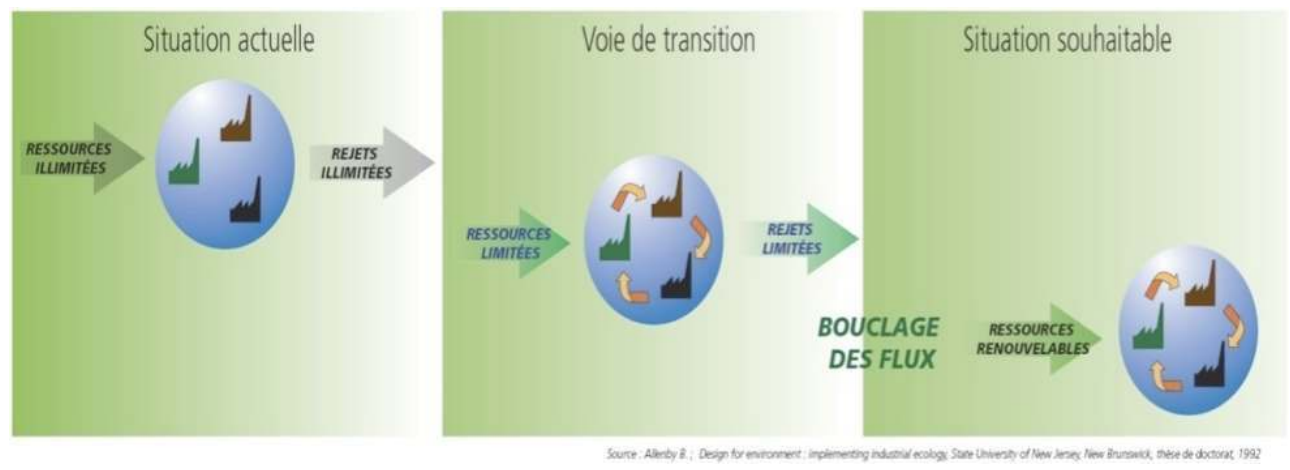

Source : Allenby, 1992

5 L'environnement et l'économie ne sont plus complètement séparées voire opposées mais interdépendantes et associées.

\section{L'écologie industrielle : le pari de la dématérialisation}

6 Cette approche systémique est par essence indispensable pour apporter des réponses efficaces à la problématique d'une incroyable complexité que nous propose le concept de développement durable (Adoue, 2004).

7 Plusieurs niveaux d'actions se dessinent, grâce à cette vision systémique proposée par l'écologie industrielle. Il est en effet possible et pertinent d'agir sur les produits, sur les systèmes de production, sur les modèles d'affaire...

Il semble toutefois important de souligner que si certains des éléments théoriques ayant contribué au diagnostic sur le fonctionnement de notre société industrielle contemporaine (limites des stocks de ressources, des capacités de la biosphère à subir les impacts humains...) (Georgescu-Roegen, 1979) sont partagés avec les tenants de la " décroissance », les outils imaginés par les animateurs de l'écologie industrielle au début des années 1990 l'ont de toute évidence été pour rendre l'économie de croissance compatible avec ces limites. Il s'agit de dématérialiser la croissance, de produire avec moins de matières et d'énergie par unité de croissance... pas de remettre en question le modèle... faute de modèle alternatif opérationnel. Cette clarification semble fondamentale pour l'examen proposé dans cet article, celui des différents outils proposés par le champ scientifique de l'écologie industrielle. En effet, la recherche de réponses à la 
problématique posée par le développement durable nous renvoie à deux directions possibles et difficilement compatibles.

9 La première direction, la plus évidente sur le plan théorique, consiste à remettre en question la principale cause du gaspillage de ressources mais qui est le moteur de la société industrielle contemporaine : l'économie de croissance. Elle présente toutefois de sérieux inconvénients à ce jour non-résolus : l'absence de modèle éprouvé et l'absence de stratégie de transition. En effet, la période de crise économique débutée en 2008 et toujours en cours à l'heure où cet article est rédigé nous alerte sur les impacts humains, notamment pour les populations les plus fragiles, d'une croissance économique ralentie... et donc sur les effets potentiels d'un brusque changement de modèle. Les interrogations de Serge Latouche concernant les maux d'une décroissance subie prennent alors toute leur valeur (Latouche, 2010).

10 La seconde, moins radicale, donc moins efficace d'un point de vue environnemental, consiste à aménager le modèle existant : rester dans une économie de croissance tout en l'aménageant afin qu'elle consomme moins de ressources et rejette moins de polluants. Elle possède évidemment de nombreuses limites. Dans un monde fini, cette économie de croissance associée à une explosion démographique jusqu'alors inconnue continue de consommer des quantités extravagantes de matières et d'énergie. Le pari de la dématérialisation de la croissance n'est donc pas de développer un nouveau modèle ... mais de ralentir la vitesse de la "machine économique " d'un point de vue de sa consommation de ressources. À défaut d'inventer une "marche arrière», cela peut permettre de diminuer la violence du choc lorsque cette machine rencontrera vraiment ses limites... ou de gagner du temps pour pouvoir inventer et développer cette «marche arrière ». Mais même cette idée peut être remise en question si l'on prend en compte la théorie de l'effet rebond (Adoue, 2007). La perspective d'une régulation des consommations de ressource en valeur absolue pourrait toutefois être envisagée. Les émissions de certains polluants $\left(\mathrm{CO}_{2}, \mathrm{SO}_{2} \ldots\right)$ ont déjà pu faire l'objet de régulations de ce type à travers les permis d'émission négociables... Aussi inimaginable que cela puisse l'être il y a 20 ans et malgré toutes les améliorations que demandent les systèmes en place, les émissions de gaz à effet de serre peuvent aujourd'hui être régulées en valeur absolue à l'échelle d'un continent. Pourquoi pas les consommations de ressources?

11 Ainsi, dans les années 1990, confrontés à une urgence que constituait l'avènement proche de catastrophes environnementales globales (réchauffement climatique), les fondateurs de l'écologie industrielle se sont focalisés sur l'aménagement du modèle existant, celui de la croissance de l'économie, avec un objectif clair : trouver des solutions opérationnelles pour la dématérialiser.

Quid du champ social?

12 La dimension sociale n'apparait que plus tard, à partir de 1996, dans le rapport Sustainable America (PCSD-president council for substainable development - Al Gore). Ce rapport concernait la création de parcs éco-industriels et mettait en avant le partage équitable des ressources humaines (apparition de l'homme). Alors qu'elle oscillait entre écologie et économie, l'écologie industrielle devient alors réellement un champ disciplinaire regroupant les trois piliers du développement durable: l'écologie, l'économie et le social. 


\section{L'écologie industrielle en France}

13 Dans le monde francophone, le suisse Suren Erkman a largement diffusé les théories existantes en matière d'écologie industrielle, et son sous-titre mettre en pratique le développement durable dans une société hyper-industrielle (Erkman, 1998) caractérise les relations entre les notions d'écologie industrielle et de développement durable. Cependant, pour lui, "l'écologie industrielle ne s'aventure pas sur le terrain de l'écologisme politique» refusant de sombrer dans le scientisme ou le catastrophisme, inhérents selon lui à ce terrain.

14 En France, c'est en 2006 que l'Agence Nationale de la Recherche organise un Atelier de réflexion prospective en Écologie Industrielle (ARPEGE) en vue d'une «mise au point conceptuelle » sur les définitions, les enjeux, les retours d'expériences et programmes de recherche engageables. Dans son rapport final, la définition commune aux membres du consortium est la suivante: "[...] L'Écologie Industrielle porte une attention particulière à l'analyse des échanges entre les sociétés et la nature et à la circulation des matières et de l'énergie qui les caractérisent ou qui caractérisent les sociétés industrielles elles-mêmes. [...] L'Écologie Industrielle constitue ainsi un champ de recherche pluri et interdisciplinaire mais aussi une démarche d'action dans la perspective d'un développement durable. [...] ». La liaison entre l'écologie industrielle et le développement durable est ainsi largement reconnue par la recherche française.

\section{L'écologie industrielle et les outils qu'elle propose}

15 L'écologie industrielle consiste en une approche systémique qui a pour objet de dématérialiser le fonctionnement de la société industrielle contemporaine et l'économie de croissance, de boucler les flux de matière et d'énergie, de décarboner l'énergie et de limiter les pertes par dissipation. Derrière ce champ et ces objectifs, il y a le développement d'un certain nombre d'approches opérationnelles.

16 Le caractère systémique amène plusieurs axes d'actions à différents niveaux. Il permet d'envisager d'agir sur différents sous-systèmes de la société industrielle en utilisant plusieurs leviers : penser des actions sur l'organisation de la production (au travers les symbioses), proposer des réorganisations de la consommation de produit et service qui est à la base des problèmes de ressources.

\subsection{Les outils de diagnostic : le métabolisme industriel}

La modélisation des métabolismes tels que précédemment définis en écologie urbaine par Odum puis Duvignaud, ou encore les travaux des professeurs Baccini et Ayres, la modélisation du système industriel et de ses interactions avec la biosphère, la comptabilité des flux de matières et d'énergie, correspondent à la colonne vertébrale de l'écologie industrielle. Cet outil destiné à l'optimisation systémique est au centre des préoccupations (figure 2). 
Figure 2 : Métabolisme urbain, métabolisme lacustre

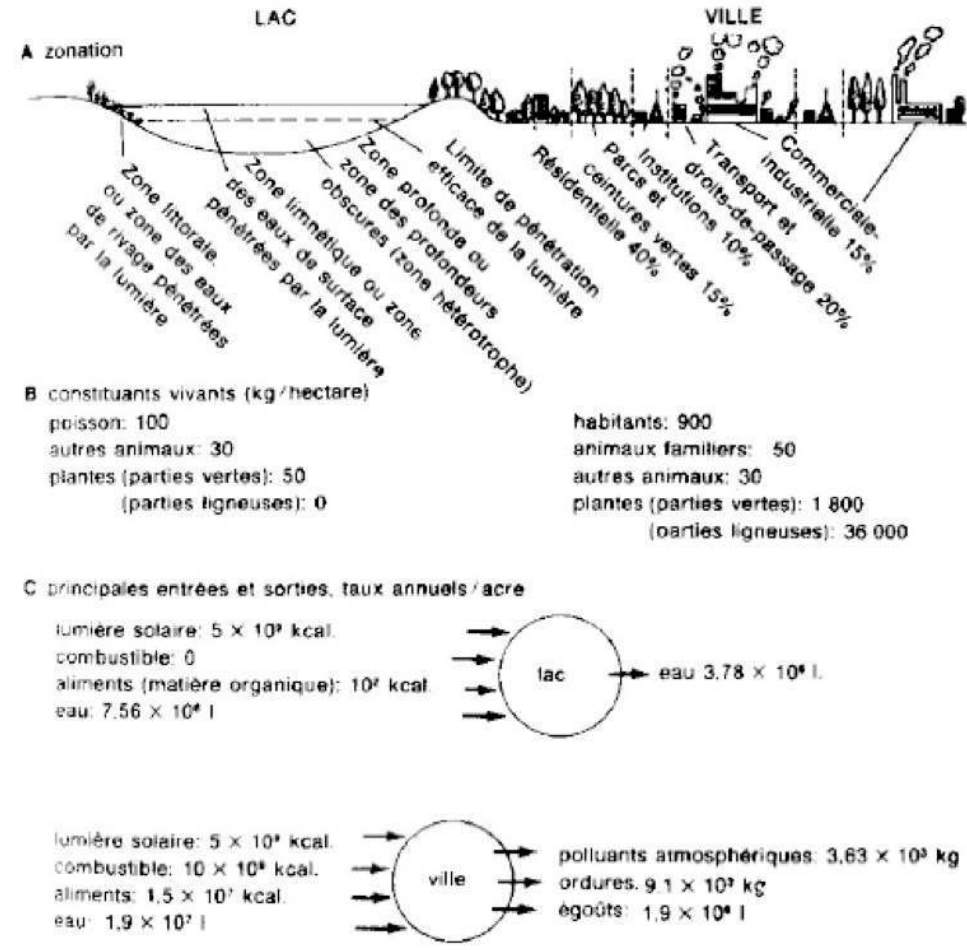

Source : E. P. ODUM, 1975, p. 39

Figure 3 : L'écosystème urbs selon Paul Duvigneaud

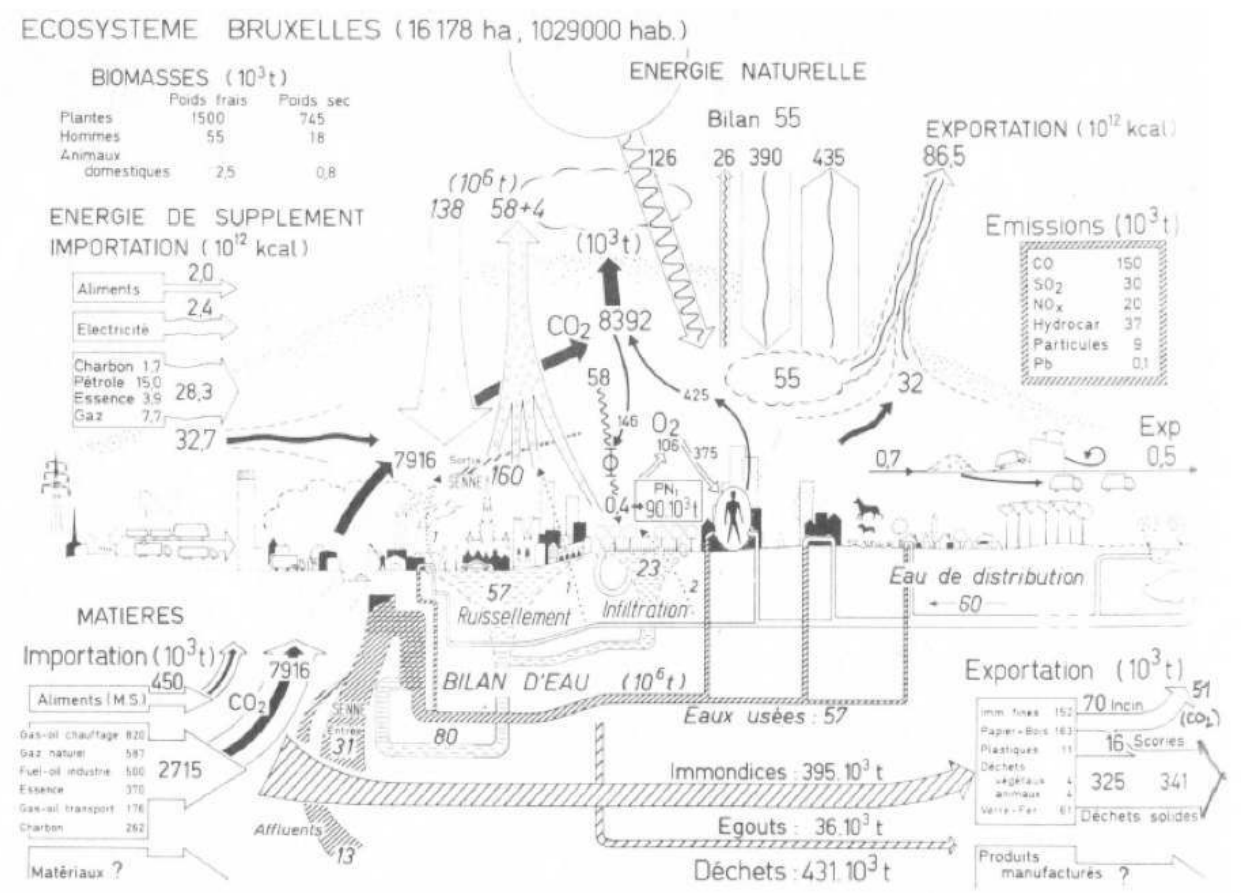

Source : Duvignaud, 1980, p. 300. 
18 À partir de ces approches de comptabilité matière/énergie d'un système antérieures à la naissance de l'écologie industrielle, ses fondateurs vont développer une famille d'outils : les outils de métabolisme industriel.

Figure 4 : Les outils de métabolisme industriel

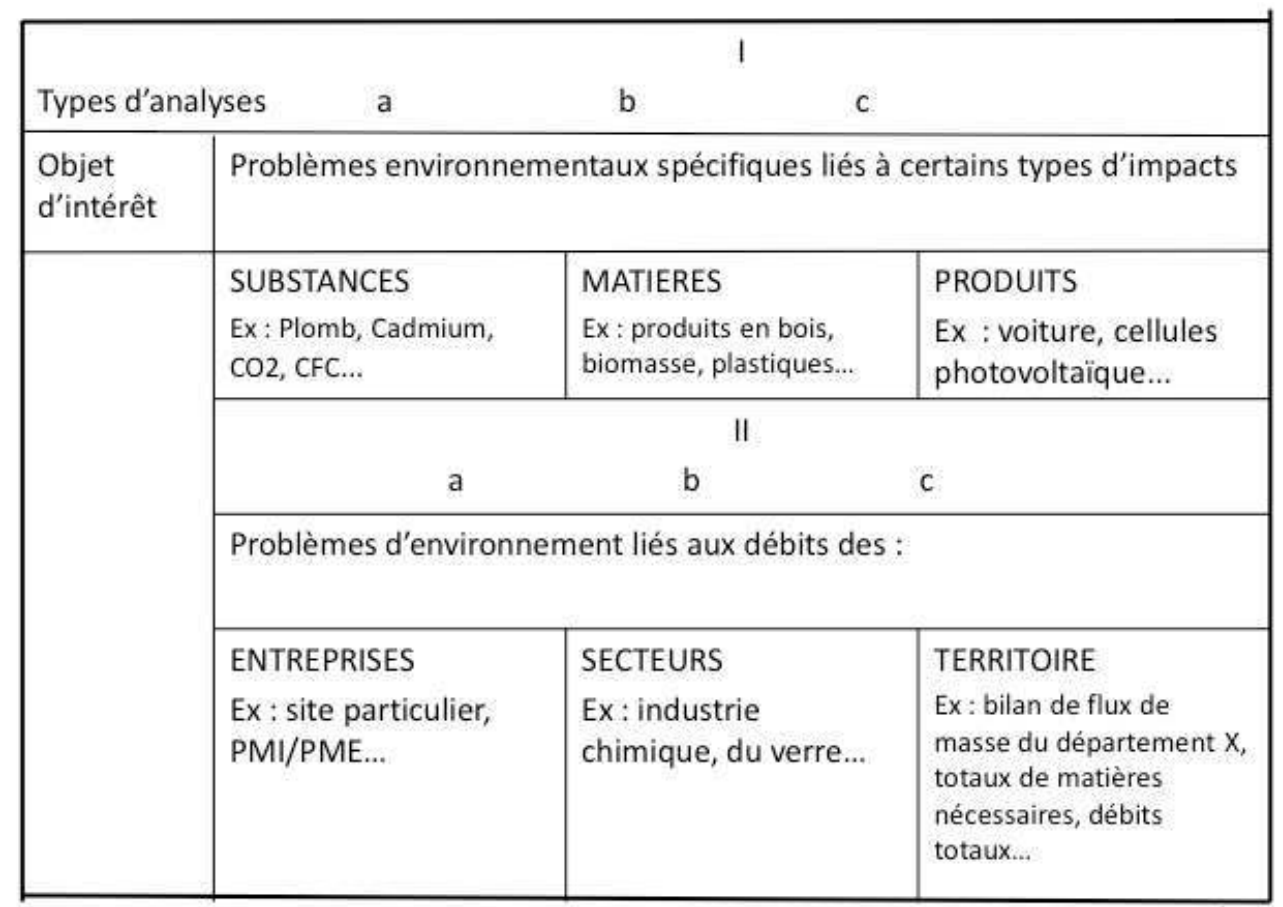

Source : Bringezu et Moriguichi (2002)

Certains sont aujourd'hui incontournables. L'analyse de cycle de vie (ACV) est par exemple une méthode qui fait l'objet d'une normalisation ISO (ISO14040).

\subsection{Les approches opérationnelles - optimiser les consommations et les rejets d'un système de production au sein d'un périmètre défini : les symbioses industrielles}

Selon la définition de Marian Chertow (Chertow, 2004), la symbiose industrielle est un sous champ de l'écologie Industrielle qui concerne principalement la gestion coopérative des flux de ressources au travers des réseaux de métiers comme un moyen d'une approche écologiquement soutenable de l'activité industrielle.

21 L'axe de travail opérationnel le plus spectaculaire de l'écologie industrielle, auquel on la réduit souvent, est donc bien celui de la symbiose industrielle. L'éco-parc dont Kalundborg en reste l'exemple toujours emblématique (Jacobsen, 2006; Nahapetiana, 2007) qui illustre le fonctionnement d'un écosystème industriel bouclé et les bénéfices qui en découlent, tant au niveau de la consommation de matière que de la réduction des déchets et de ses avantages financiers. Quelle thèse sur le sujet n'évoque pas Kalundborg? 


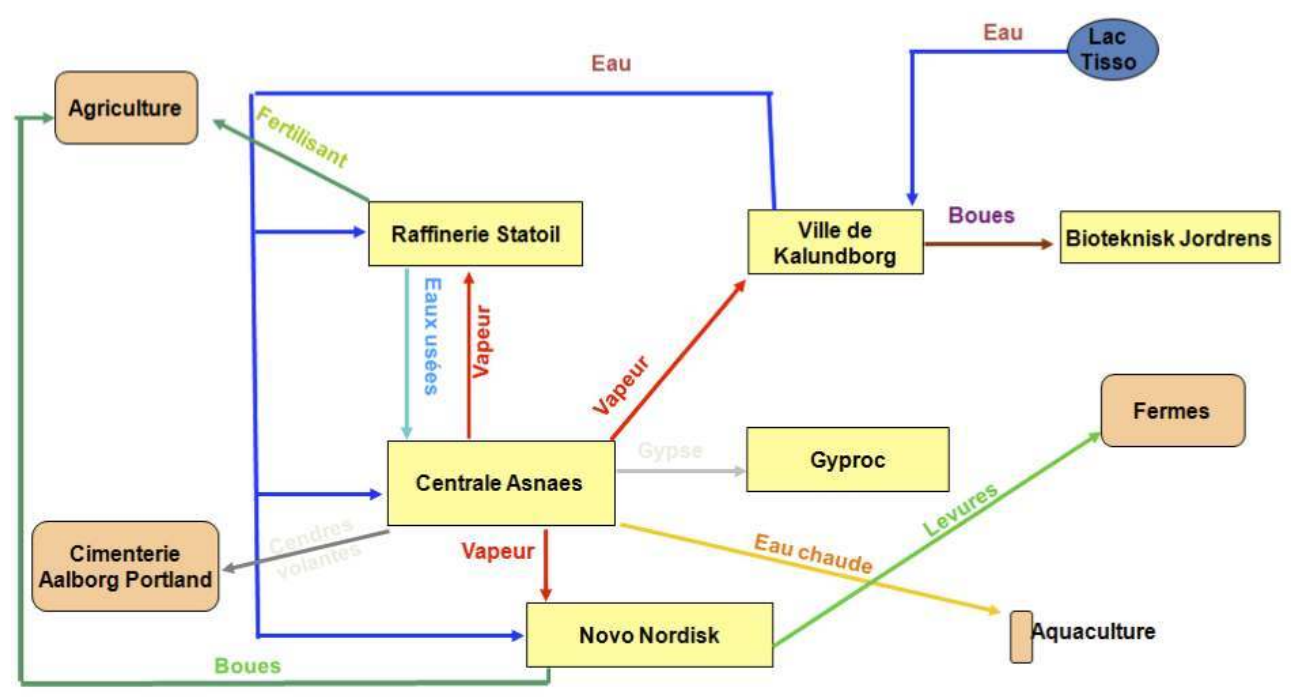

Source : Jacobsen, 2006, p. 242 périmètre géographiquement délimité entre toutes les entités qui y sont inscrites en faisant abstraction des filières établies.

\subsection{Agir sur le produit : l'éco-conception}

Le deuxième axe pour dématérialiser le fonctionnement de l'économie de croissance consiste à agir sur l'élément clé dans la demande de ressources (nécessaire à sa fabrication) et dans la production de déchets : le produit, et par extension, le service. La conception des biens et services présente donc toutes les caractéristiques des leviers opérationnels recherchés par les fondateurs de l'écologie industrielle. L'éco-conception va ainsi se développer. Cette éco-conception est clairement dans le champ de l'écologie industrielle dans ses premières années. En effet, Allenby théorise dans la première partie de sa thèse l'écologie industrielle et propose dans la deuxième partie une méthode d'écoconception. L'éco-conception s'est aujourd'hui émancipée de la sphère de l'écologie industrielle. Elle est un sujet mature que les industriels et les législateurs se sont appropriés car elle se situe dans le prolongement des pratiques classiques. Elle est le levier proposé par l'écologie industrielle qui s'est le plus développé et qui est le plus couramment utilisé. Sa normalisation (ISO 14062) a grandement participé à cette appropriation de même que les diverses réglementations européennes (directive sur les véhicules hors d'usage, consommation électriques, IPPC).

Ce deuxième axe concentré sur le produit (ou le service), est cantonné aux frontières des entreprises et de leurs fournisseurs qui la pratiquent, de façon plus ou moins contrainte par la règlementation. De ce fait, cette pratique n'est pas définie sur un périmètre territorial mais plutôt sectoriel. 


\subsection{Agir sur les modèles d'affaires : l'économie de la fonctionnalité}

Le troisième levier consiste à agir sur le modèle commercial, le business model, c'est à ce niveau que se positionne l'économie de la fonctionnalité. L'économie de la fonctionnalité est présente dans l'écologie industrielle dès 1994. W. Stahel et 0 . Giarini la définissent dès 1986, mais l'écologie industrielle va devenir son «vase d'expansion ». L'économie de la fonctionnalité propose de commercialiser le service rendu en lieu et place du produit. Elle bouleverse en cela les logiques industrielles classiques: en schématisant, produire en grandes quantités, dans des pays à bas coûts de main-d'œuvre souvent éloignés et entretenir la demande, notamment à partir de l'obsolescence programmée des produits. Avec l'économie de la fonctionnalité, la marge de l'industriel va se définir sur d'autres critères : la durabilité des biens assurant le service vendu, la diminution des entrants et des sortants liés à la fourniture de ce service. Il s'agit d'un bouleversement considérable : les logiques économiques changent, les métiers également. Il faut en effet assurer la maintenance des biens, leur remanufacturing. S'il s'agit d'un produit énergétique (exemple: vendre une température ambiante à la place d'un kwh), il faudrait même interroger d'autres secteurs d'activité (bâtiment...).

Si, par la nature radicale de ce qu'elle implique pour les logiques industrielles, l'économie de fonctionnalité se présente comme un des leviers les plus pertinents proposés par l'écologie industrielle, elle pourrait avoir du mal à se développer. Ces bouleversements peuvent en effet effrayer les entrepreneurs, d'autant plus si l'entreprise qui doit évoluer vers l'économie de fonctionnalité est de taille importante.

\section{Conclusion : Différents leviers, différents niveaux d'action mais une grande complémentarité}

Ce nouveau levier que constitue l'économie de fonctionnalité se justifie par la nature systémique de l'écologie industrielle. C'est le rôle de l'écologie industrielle de continuer à traiter ces sujets de façon coordonnée. Le rapport de l'ARPEGE revendique l'économie de la fonctionnalité parmi les "différentes stratégies» de l'écologie industrielle. Ce troisième axe ne présente pas de caractéristiques territoriales. 
Figure 6 : Frontières et complémentarité des outils opérationnels de l'écologie industrielle

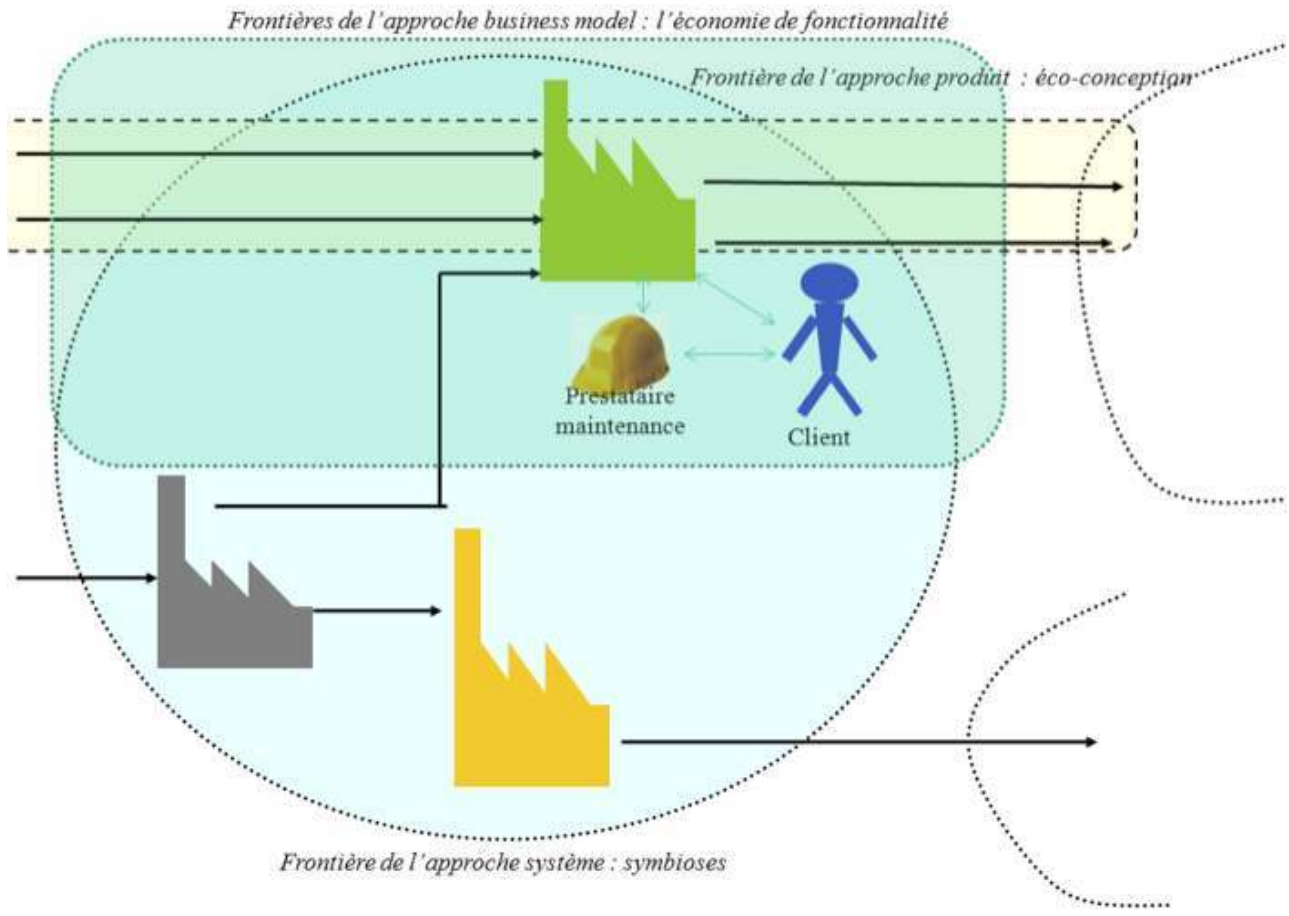

Ces trois axes opérationnels sont bien les principales déclinaisons du champ scientifique que constitue l'écologie industrielle.

Dans une optique de dématérialisation, symbioses, éco-conception et économie de la fonctionnalité doivent se confronter aux mêmes questions concernant leur efficacité, leur éco-efficience : constituent-elles des solutions pour que la consommation de ressources soit minimale en rapport au PIB ? Les changements qu'elles induisent sont ils acceptables par les organisations et leurs clients et fournisseurs ? Comment le législateur peut-il coordonner l'utilisation de ces différents leviers?

Adaptée à la compréhension de la complexité, orientée vers la dématérialisation de l'économie, l'écologie industrielle peut, dans notre modèle de société de consommation, proposer a minima des optimisations. Mais dans un monde de pénuries croissantes, ne sera-t-elle pas une voie par assurer notre subsistance?

\section{BIBLIOGRAPHIE}

Adoue C., 2004, Méthodologie d'identification des synergies éco-industrielles réalisables entre entreprises sur le territoire français, Thèse de doctorat en écologie industrielle. Troyes : UTT, 2004, 224 pages.

Adoue C., 2007, Mettre en œuvre l'écologie industrielle, Lausanne, Presses polytechniques et universitaires romandes, 106 pages. 
Allenby B., 1992, Design for environnement: implementing industrial ecology, thèse de doctorat en écologie industrielle, University of New Jersey, New Brunswick, 381 p.

Chertow M., Ashton W., Kuppalli R., 2004, The Industrial Symbiosis Research Symposium at Yale: Advancing the Study of Industry and Environment, Yale school of forestry \& environmental studies, Report $\mathrm{n}^{\circ}$ 3, Yale Publishing Services Center, $41 \mathrm{p}$.

Duvigneaud P., 1980, La synthèse écologique. Populations, communautés, écosystèmes, biosphère, noosphère, $2^{2 \mathrm{ème}}$ éd. [1 $1^{\text {ère }}$ éd. 1974], Paris, Doin.

Erkman S., 1998, Vers une écologie industrielle, éditions Charles Léopold Mayer.

Frosch R., Gallopoulos N., 1989, "Strategies for Manufacturing", Scientific American, Septembre, p. $144-152$.

Georgescu-Roegen N., 1979, La décroissance : Entropie - Écologie - Économie, Chicoutimi : J.-M. Trembla.

Jacobsen, N.B, 2006, "Industrial symbiosis in Kalundborg, Danemark: A quantitative assessment of economic and environmental aspects", Journal of Industrial Ecology 10 (1-2), p. 239-255.

Latouche S., Harpagès D., 2012, Le temps de la décroissance, Le Bord de l'eau, coll. « Troisième culture », $92 \mathrm{p}$.

Nahpetian N., 2007, L'écologie industrielle : la symbiose industrielle de Kalundborg, Belgique : Lessius Hogeschool. Cas d'étude - Syllabus de français Ba 2.

Odum E.P., 1975, Écologie : un lien entre les sciences naturelles et les sciences humaines, traduction de la $2^{\text {ème }}$ éd. de Ecology, 1975 (1 $1^{\text {ère }}$ éd. 1963), Paris, Doin.

\section{AUTEURS}

\section{CYRIL ADOUE}

Cyril Adoue est ingénieur en génie des systèmes industriels et docteur en études environnementales (il a produit une des premières thèses françaises en écologie industrielle). Il est l'auteur de l'ouvrage Mettre en œuvre l'écologie industrielle (2007, Presses Polytechniques et universitaires romandes, 106 p). Il a participé à l'accompagnement de projets et de démarches pour de nombreux territoires, en s'intéressant notamment à la question du métabolisme (notamment dans le cadre de la mise en œuvre du logiciel Prestéo), mais aussi des conditions socio-économiques et du jeu d'acteurs.

\section{LAURENT GEORGEAULT}

Laurent Georgeault est ingénieur en systèmes d'information et concepteur du logiciel Prestéo. Il accompagne les entreprises et les territoires dans la mise en œuvre de l'écologie industrielle et produit des outils spécialisés dans l'aide à la décision pour l'EI, la détection de symbioses, la construction de métabolismes et l'évaluation environnementale. 